\title{
Investing in the future
}

\author{
Nanotechnology is an important tool in the fight against malaria.
}

In 2013, over 200 million cases of malaria were reported worldwide; in the same year, over 650,000 people died from the disease, most of them children ${ }^{1}$. Yet malaria is preventable and treatable. The reason it persists is because of limitations in funding, logistics and technology, as well as the increasing resistance of the disease to antimalarial drugs. Malaria, which is spread by female Anopheles mosquitos infected with Plasmodium parasites, is inextricably linked to poverty, with the highest mortality in developing countries. World Malaria Day ${ }^{2}$ will take place later this month on 25 April and its aims align with the United Nations Millennium Development Goals ${ }^{3}$, which were set in 2000 and include targets to dramatically reduce poverty and disease by 2015 . Since 2013, World Malaria Day has operated under the theme 'Invest in the future: defeat malaria', and nanotechnology is one of the weapons in this fight.

The use of nanoparticles in rapid diagnostic tests was introduced in the 1990s and has since improved our understanding of the prevalence of the disease. Increased access to these tests, which yield a simple yes/no answer, has also improved the rate of therapeutic intervention. (World Health Organization (WHO) guidelines advise that treatment is only administered after a positive malaria test in a bid to avoid the non-discriminant prescription of antimalarials and to slow down the rate of resistance.) Many of these rapid diagnostic tests are based on antibody-labelled gold nanoparticles that are specific for lysed malarial antigens. If the malaria parasites are present, the labelled nanoparticles bind to them and are arrested on the test strip, forming a band that can be seen by the naked eye and indicating a positive result. The beauty of these tests is their simplicity. Unlike the current standard test for malaria (microscopic examination of blood films) very little sample is needed - just a drop of blood that can be taken from a finger prick. Furthermore, the tests require little specialist training, chemicals or equipment, and the results are obtained in minutes, as opposed to days. However, these tests are not perfect and inconsistencies in their manufacture can lead to discrepancies in sensitivity and false-positives. This means that the WHO regularly evaluates these rapid diagnostic tests to inform users of the reliability of their performance ${ }^{4}$.

Recent developments in nanotechnology have attempted to overcome some of the problems currently facing malarial diagnostics. For example, Dmitri Lapotko and co-workers have developed a needlefree method of diagnosing malaria ${ }^{5}$. Malaria parasites can form nanoparticles, known as hemozoins, in red blood cells, and using laser pulses, the researchers generate vapour bubbles from the hemozoins. When these bubbles burst, an acoustic signal generated only by these nanoparticles can then be quickly detected. The researchers are hopeful that this sensitive and specific technology can be converted into a portable device for use in the field, with a single test costing as little as $50 \mathrm{US}^{\mathrm{u}}$ cents $^{6}$.

The nanotechnology community is also working to counteract the increase in antimalarial resistance by improving the accuracy of diagnosis. Using components bought on eBay, Jonathan O'Halloran developed a nanowire-based biosensor that can read DNA directly from blood samples. The company QuantuMDX was subsequently established and, over the past six years, has worked to develop the technology into a handheld point-ofcare device for comprehensive malaria diagnostics. The technique requires a drop of blood loaded into a cassette that isolates the malarial DNA. The DNA is then amplified and sent to a biosensor array containing 100 to 1,000 nanowire fieldeffect transistors, each one functionalized to detect different malarial strains, subtyping and resistance markers. The device provides a readout within 15 minutes, detailing the species of parasite and its potential for drug resistance. This information can then be used to create a course of treatment that is personally tailored to counter the resistance. Furthermore, the information generated can be shared using a mobile data connection, contributing to the epidemiological profile of the disease and its strains.

Longer-term solutions to malaria, such as vaccines, are still to be fully realized. At present, there is no commercially available malaria vaccine, although it is hoped that one candidate - a vaccine known as RTS, $S$ - could be approved by next year ${ }^{7}$. Currently in phase III trials, this vaccine is over five years ahead of the closest vaccine candidates in terms of development; however, with an efficacy of around 55\% in the reduction of malaria episodes in the year following vaccination, it is regarded as an additional strike against malaria and not a replacement for existing interventions. $R T S, S$ does not use a nanoparticle formulation. However, the stability that can be conferred to therapeutics by using a nanomaterial platform could potentially improve the performance of such vaccines. It could also increase the targeting and efficacy of vaccines at low doses.

Overall, it is imperative that the assault on malaria remains constant; the ability of the disease to proliferate, particularly during the rainy season, can quickly reverse any progress. This requires pragmatic solutions to problems such as the logistics of distributing interventions and continuous funding for research into effective and long-lasting prophylaxis and treatments. Although the WHO reports that research funding for malaria has increased from less than US\$100 million in 2000 to almost US $\$ 2$ billion in 2012, this is still less than the US $\$ 5.1$ billion that the WHO says is required annually to eradicate malaria ${ }^{1}$. Notably, scientists are increasingly looking to internet crowd-funding campaigns to support their research. By enabling the public to define the spending of research budgets, funds can be driven towards what society perceives as the most pressing challenges. QuantuMDx has, for example, recently launched its own campaign to accelerate the development and trialling of its diagnostic device in Africa, with a target of US $\$ 50,000^{8}$. As the fund climbs towards its target, it seems as though both researchers and the public believe that nanotechnology has a role to play in defeating malaria.

References

1. http://www.who.int/malaria/publications/world_malaria report_2013/report/en/

2. http://www.worldmalariaday.org/home_en.cfm

3. http://www.un.org/millenniumgoals

4. http://www.who.int/tdr/publications/rapid_diagnostic/en/

5. Lukianova-Hleb, E. Y. et al. Proc. Natl Acad. Sci. USA 111, 900-905 (2014)

6. http://www.nytimes.com/2014/01/07/science/a-new-test-formalaria-no-blood-required.html

7. http://www.who.int/immunization/research/development/ WHO_malaria_vaccine_q_and_a_Oct2013.pdf

8. http://www.indiegogo.com/projects/the-future-quantumdx-shandheld-dna-lab 